\title{
COBALT NANOPARTICLES WITH TUNED INTERACTION POTENTIAL: TOWARDS WORM- AND CHAIN-LIKE AGGREGATES
}

\author{
C. Dobbrow ${ }^{1}$, M. Vaidyanathan ${ }^{1,2}$, L. Belkoura ${ }^{1}$, \\ D. Guin ${ }^{1}$, A.M. Schmidt ${ }^{1 \dagger}$ \\ 1 Department Chemie, Universität zu Köln, Luxemburger Str. 116, D-50939 Köln \\ ${ }^{2}$ National Institute of Technology, Dumas Road, Ichchhanath Surat-395007, India \\ ${ }^{\dagger} e$-Mail: annette.schmidt@uni-koeln.de
}

1. Introduction. The process of self-assembly of molecular and nanoscopic objects by organization into highly integrated and functional structures is well known in nature and plays a crucial role for biological materials. Similarly, the nanolevel structuring in synthetic materials is of great impact on mesoscopic features and also on the macroscopic properties, however, the artificial realization of nanolevel self-assembly still remains scarce [1-2].

While synthetic chemistry currently experiences a fast forward in the design of nanoscale structures, it remains a challenge to translate the respective interactions towards a controlled arrangement with tuned mesoscopic, e.g., anisotropic properties [3-7]. Optimally, molecules and nano objects self-assemble by spontaneous materialization into organized aggregates or networks. Various interactive mechanisms play an important role in this process, such as electrostatic and dipolar interactions, surface properties and the interaction of surface-attached mediating agents [8].

For the alignment of anisotropic nano and micro objects and for their organization into two-dimensional chain-like structures, the magnetic dipoles of ferromagnetic particles are of high interest. Cobalt-based nanoparticles are shown to assemble into well-defined nanoscopic chains, first reported by Pyun et al. for polymer-coated ferromagnetic particles, which form necklace-like structures [910]. Similar particles are reported to form rings under certain conditions, being influenced by the magnetic particle concentration, their interaction, chain stiffness and thermal fluctuation of the particles [11-13]. The ring structure can be assumed to be the energetically favored state in the $2 \mathrm{D}$ level of such ensembles [14]. However, the chain and ring structures are mainly observed by transmission electron microscopy of drop-casted samples. As thus they are influenced by processes during drying; conventional TEM structure investigations do not allow to investigate equilibrium structures in dispersion and to systematically examine property-structure relationships.

In this work, we use intrinsic particle properties to create a balance between repulsive and attractive forces in ferromagnetic particles. This opens a pathway to the design of super-structured ferromagnetic particle ensembles from the interplay of attractive dipole-dipole interaction and repulsive electrostatic forces. We vary the amount of charge-inducing ligands, tri-octylphosphine oxide (TOPO) during the particle synthesis and investigate the influence of the TOPO concentration 
on the particle size distribution and core self-assembly, both by TEM and by dispersion-based methods. Unexpected correlations between the core size, hydrodynamic radius and the magnetic properties are found, and the evolving structures can be tailored by adjusting the synthetic conditions in terms of chain morphology and degree of order.

\section{Experimental part.}

2.1. Materials and instrumentation. Dicobalt octacarbonyl $\left(\mathrm{Co}_{2}(\mathrm{CO})_{8}\right.$, $95 \%$ with $5 \%$ hexane as a stabilizer) is purchased from Sigma-Aldrich. 1,2Dichlorobenzene (DCB, anhydrous, 99\%) and dichloromethane (DCM) are obtained from Aldrich. Tri-octylphosphine oxide (TOPO, 99\%) is used as received from Acros Organics. $\alpha$-Bromoisobutyric acid, 2,2-bipyridyl and dimethylformamide (DMF) are purchased from Aldrich and used as received. Copper (I) bromide $(\mathrm{Cu}(\mathrm{I}) \mathrm{Br})$ is obtained from Aldrich and purified by stirring in glacial acid over night. Styrene is purchased from Aldrich and it is passed through a column of neutral alumina for purification.

Transition Electron Microscopy (TEM) images are performed on samples prepared by drop-casting on a carbon-coated copper grid, by using a Zeiss LEO 912 Omega, Oberkochen, Germany. The core size and the chain length of the particle are determined via image analysis with help of the "measure IT" software (Olympus). Dynamic Light Scattering (DLS) measurements are performed with a High-Performance Particle Sizer HPP5002 (Malvern Instruments) in toluene at $25^{\circ} \mathrm{C}$, using $1 \times 1 \mathrm{~cm}^{2}$ quartz glass cuvettes. Quasi-static magnetometry is implemented on an ADE Magnetics Vibrating Sample Magnetometer (VSM) EV7. AC susceptometry experiments are performed on a DynoMag (Imego, frequency range $1 \mathrm{~Hz}-250 \mathrm{kHz}$ ). The field amplitude amounts to $5 \mathrm{kA} / \mathrm{m}$ in the frequency range up to $1 \mathrm{kHz}$ and decreases continuously to $0.5 \mathrm{kA} / \mathrm{m}$ between $1 \mathrm{kHz}$ and $250 \mathrm{kHz}$. Elemental analysis (EA) of carefully washed and dried particles is carried out with an Elementar Vario EL (Elementar). Gel Permeation Chromatography (GPC) are performed in tetrahydrofurane (THF) from a system with a column combination (Latex/styragel 50/1000 nm pore size) and on-line UV detector 481. The system is calibrated versus commercially available polystyrene standards.

2.2. Synthesis. All synthetic procedures were performed under argon atmosphere.

Synthesis of carboxylic acid-telechelic polystyrene. Carboxylic-acid terminated polystyrene is prepared by Atom Transfer Radical Polymerization (ATRP). $0.9 \mathrm{~g}(6.3 \mathrm{mmol}) \mathrm{Cu}(\mathrm{I}) \mathrm{Br}, 1.0 \mathrm{~g}(6.1 \mathrm{mmol}) \alpha$-bromoisobutyric acid and $1.8 \mathrm{~g}$ (11.6 mmol) 2,2-bipyridyl are placed into a Schlenk flask and degassed with argon. $5 \mathrm{ml}$ of degassed DMF is added and stirred until a red complex is formed. $60.0 \mathrm{ml}(614 \mathrm{mmol})$ of degassed styrene is added and the reaction flask is placed into an oil bath with a temperature of $110^{\circ} \mathrm{C}$ for 48 hours. The reaction mixture is diluted in $300 \mathrm{ml}$ DCM and passed through an alumina column to remove the copper catalyst. The obtained polymer solution is concentrated and precipitated into methanol. The polymer is vacuum dried and a white powder is obtained, which is subjected to GPC analysis $\left(\mathrm{M}_{n}=6100 \mathrm{~g} / \mathrm{mol}, \mathrm{M}_{\mathrm{W}}=8200 \mathrm{~g} / \mathrm{mol}\right)$.

Synthesis of Co@PS/TOPO nanoparticles. The preparation of cobalt particles stabilized by carboxylic acid-telechelic polystyrene and TOPO is performed by thermolysis of $\mathrm{CO}_{2}(\mathrm{CO})_{8}$ in the presence of the stabilizers. Therefore, $0.80 \mathrm{~g}$ $(2.30 \mathrm{mmol})$ of $\mathrm{Co}_{2}(\mathrm{CO})_{8}$ is dissolved in $8.0 \mathrm{ml}$ of $\mathrm{DCB}$ at ambient temperature, and the resulting solution is rapidly injected into a solution of $0.40 \mathrm{~g}(6.67$. $10^{-2} \mathrm{mmol}$ ) of carboxylic acid-telechelic polystyrene in $12 \mathrm{ml}$ of DCB pre-heated to $T=180^{\circ} \mathrm{C}$ in the presence of various amounts of TOPO ranging from zero to 


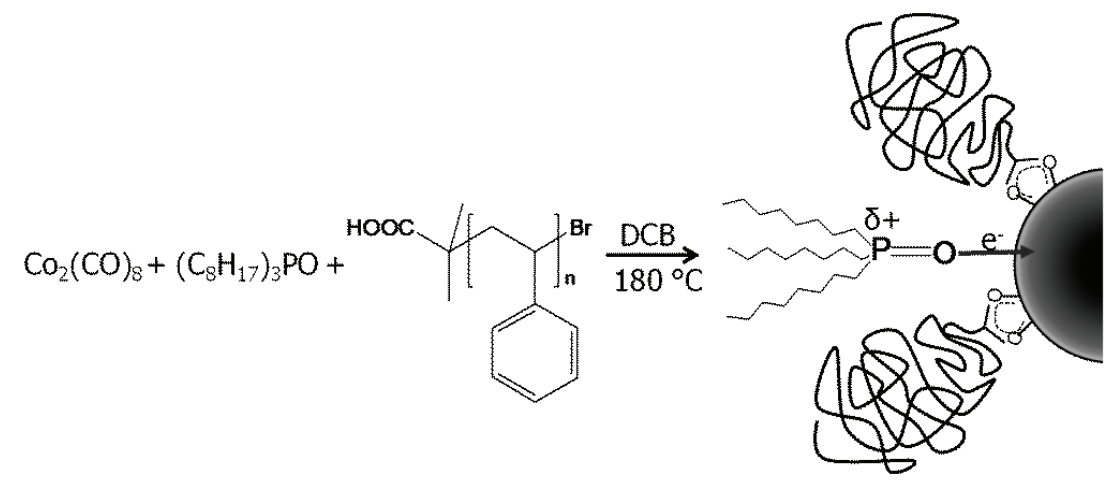

Fig. 1. Reaction scheme for the synthesis of Co@PS/TOPO particles.

Table 1. Synthetic conditions and resulting properties of Co@PS/TOPO particles synthesized at different TOPO concentrations. $c_{\text {TOPO }}$ - concentration of TOPO during synthesis; $d_{\mathrm{c}}-$ number-average core diameter as obtained by TEM; $D_{\mathrm{t}}$ - diffusion coefficient from DLS; $f_{\max }$ - peak frequency in AC susceptometry.

\begin{tabular}{ccccc}
\hline \hline Sample & $c_{\text {TOPO }}[\mathrm{mmol} / \mathrm{l}]$ & $d_{\mathrm{c}}[\mathrm{nm}]$ & $D_{\mathrm{t}}\left[\mu \mathrm{m}^{2} / \mathrm{s}\right]$ & $f_{\max }[\mathrm{Hz}]$ \\
\hline Co-0T & 0 & 17.5 & 17.8 & 83 \\
Co-6.3 & 6.3 & 22.4 & 21.4 & 18700 \\
Co-12.7T & 12.7 & 28.0 & 34.3 & 6900 \\
Co-25.4T & 25.4 & 29.4 & 41.6 & 6600 \\
\hline \hline
\end{tabular}

$0.20 \mathrm{~g}(0.52 \mathrm{mmol})$ (see Table 1$)$. After $30 \mathrm{~min}$ of heating, the reaction mixture is cooled down to ambient temperature. The particles are washed with hexane several times, and the produced particles are redispersed in toluene. The particle dispersions show black color and a high dispersion stability.

3. Results and discussion. In order to investigate the interplay between attractive and repulsive forces in the structure formation of ferromagnetic particles, we produced cobalt nanoparticles by thermal decomposition of $\mathrm{Co}_{2}(\mathrm{CO})_{8}$ in the presence of carboxylic acid-telechelic polystyrene and TOPO (Fig. 1). While the linear polymer with a chain length $\left(M_{n}\right)$ of $6100 \mathrm{~g} / \mathrm{mol}$, serving as the predominant steric stabilizer in the evolving particle dispersion, is kept at constant concentration in all batches, the TOPO concentration is varied. The resulting effect on the particle size and self-assembly has been thoroughly studied by electron microscopy, magnetic methods and scattering techniques.

By TEM analysis of the respective particle batches, we find a variation in the geometrical size of the highly contrasting particle cores increasing from $17 \mathrm{~nm}$ to $30 \mathrm{~nm}$ with the increasing TOPO content (Table 2). While at low TOPO concentrations, predominantly spherically shaped Co particles are found, the application of high TOPO concentrations results in the formation of cubic particles (Fig. 2).

Beyond the particle size and shape, the TEM images reveal an unexpected trend of the different particle batches with respect to the self-organization of the particles. An increase in particle size is generally assumed to be related to an increased magnetic moment. Therefore, an increased contribution of the attractive particle-particle interaction is expected for the larger particles. In contrast to 

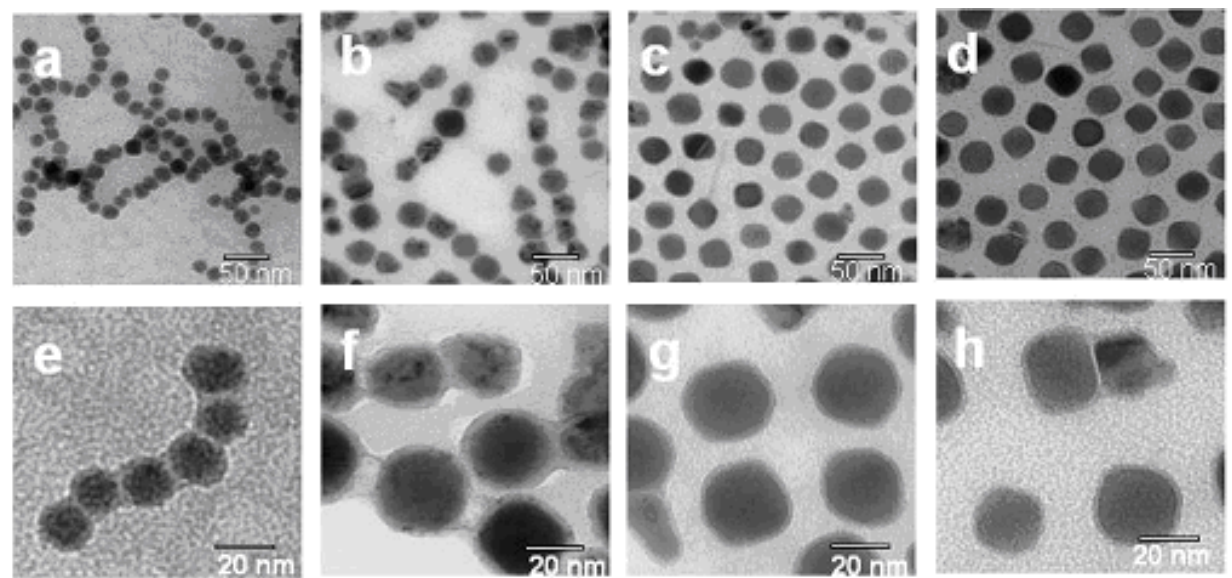

Fig. 2. TEM images of Co@PS/TOPO particles from different batches: $(a)$ and $(e)$ Co-0T; $(b)$ and $(f)$ Co-6.3T; $(c)$ and $(g)$ Co-12.7T; $(d)$ and $(h)$ Co-25.4T (see Table 1 for sample specification).

Table 2. Magnetic properties of Co@PS/TOPO nanoparticle powders. $M_{\mathrm{S}}$ - saturation magnetization, $M_{\mathrm{R}}$ - remanence magnetization, $H_{\mathrm{c}}$ - coercitivity field strength.

\begin{tabular}{ccccc}
\hline \hline Sample $[\mathrm{dry}]$ & $c_{\mathrm{TOPO}}[\mathrm{mmol} / \mathrm{l}]$ & $M_{\mathrm{S}}\left[\mathrm{Am}^{2} / \mathrm{kg}\right]$ & $M_{\mathrm{R}} / M_{\mathrm{S}}$ & $H_{\mathrm{c}}[\mathrm{A} / \mathrm{m}]$ \\
\hline Co-0T & 0 & 51.5 & 0.22 & 21.1 \\
Co-6.3 & 6.3 & 47.3 & 0.35 & 28.1 \\
Co-12.7T & 12.7 & 35.0 & 0.34 & 22.2 \\
Co-25.4T & 25.4 & 31.7 & 0.34 & 12.1 \\
\hline \hline
\end{tabular}

this, we observe a severe chain formation in the sample with the smallest core size $(\mathrm{Co}-0 \mathrm{~T})$, while large particles of around $30 \mathrm{~nm}$ show hexagonal or statistical organization on TEM images of drop-casted samples (Figs. $2 a-f$, and Fig. $3 b$ ).

In order to learn more about the origin and the dynamic nature of the chain formation, we performed dynamic light scattering (DLS) and AC susceptometry experiments based on particle dispersions in toluene. At DLS, the translational diffusion coefficient $D_{\mathrm{t}}$ is extracted from the correlation function by the CONTIN method. As shown in Table $1, D_{\mathrm{t}}$ steadily decreases in the direction Co-0T Co-24.5T, indicating a faster diffusion related to a smaller effective hydrodynamic diameter for the equivalent sphere, as shown in Fig. $3 a$. A good accordance between the core diameter $d_{\mathrm{c}}$ from TEM and the hydrodynamic diameter $d_{\mathrm{h}}$ from DLS is observed only for particles produced at a high TOPO concentration.

The results are readily confirmed by AC-susceptometry experiments (see Fig. 4). The dispersed particles are exposed to an external alternating magnetic field with a frequency varying between $1 \mathrm{~Hz}$ and $250 \mathrm{kHz}$. In case of Co-0T, we observe a resonance frequency $f_{\max }$ at $83 \mathrm{~Hz}$, whereas sample Co-6.3T shows a shift of the resonance frequency to $18700 \mathrm{~Hz}$, indicating a faster rotational mobility (high rotational diffusion coefficient $D_{\mathrm{r}}$ ) of the predominantly ferromagnetic nano objects. Thus, both dynamic methods indicate a diffusion behaviour that is explainable by the formation of bigger aggregates in dispersion of Co-0T and C-6.3T, similar to those observed in TEM. More detailed analysis with respect to the geometrical object size, by means of static light scattering and depolarized dynamic light scattering, is the subject of ongoing experiments. 
Cobalt nanoparticles with tuned interaction potential:
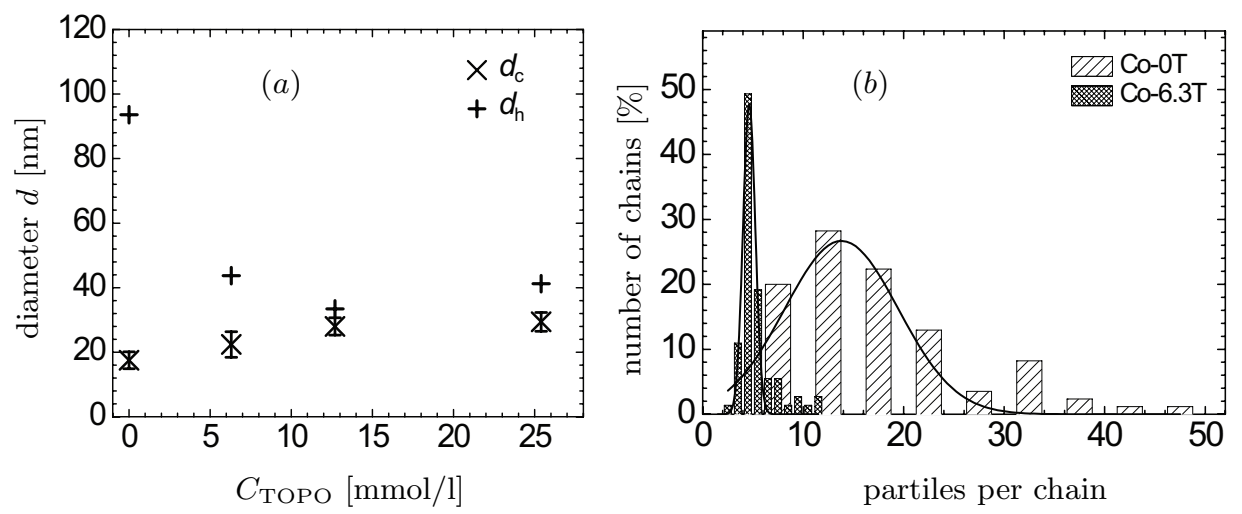

Fig. 3. (a) Core diameter $d_{\mathrm{c}}$ (from TEM) and hydrodynamic diameter $d_{\mathrm{h}}$ (from DLS) of Co@PS/TOPO particles in dependence on the TOPO content; $(b)$ chain length histogram of particle batches Co-0T and Co-6.3T.

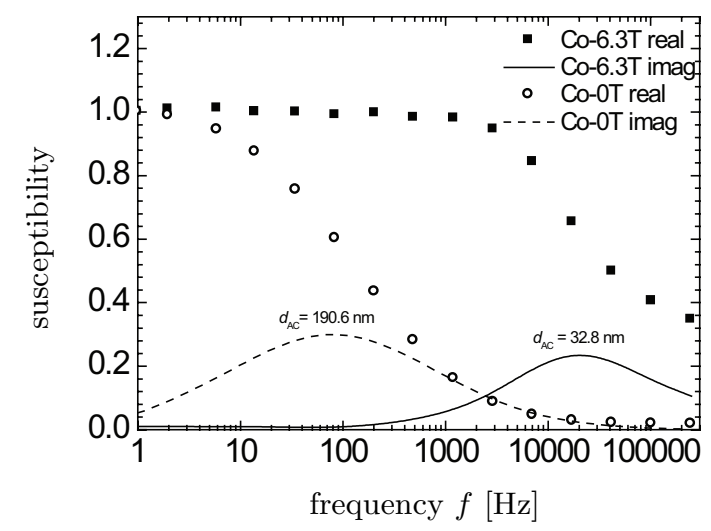

Fig. 4. AC susceptometry spectra of Co@PS/TOPO nanoparticle dispersions Co-6.3T and $\mathrm{Co}-\mathrm{OT}$ in toluene.

The static magnetic properties of the particle dispersions and of washed and dried powders are determined in quasi-static Vibrating Sample Magnetometry (VSM) experiments by recording the sample magnetization versus the external magnetic field strength.

The magnetization curve of the Co-0T powder (Fig. $5 a$ ) shows a pronounced hysteresis, typical for ferromagnetic single-domain nanoparticle powders, and a saturation magnetization $M_{\mathrm{S}}$ of up to $51.5 \mathrm{Am}^{2} \mathrm{~kg}^{-1}$. With the increasing TOPO amount at synthesis, $M_{\mathrm{S}}$ decreases, indicating an enlargement of non-magnetic components in the dried powder. In the same direction, we observe an increase in the relative remanence $M_{\mathrm{R}} / M_{\mathrm{S}}$, while the coercivity $H_{\mathrm{c}}$ is nearly constant (see Table 2).

In contrast to the powders, the magnetization graphs of the particle dispersions show no hysteresis (Fig. $5 b$ ), in accordance with the magnetization reversal occurring readily due to Brownian rotation of the well-dispersed particles. However, we observe an unexpected shape of the graph with a so-called wasp-waist anomaly. This anomaly, which is probably caused by the tendency to form chains, is more prominently observed in the variation graph $\left(M_{\mathrm{up}}-M_{\mathrm{down}}\right.$, Fig. $\left.5 c\right)$. The 


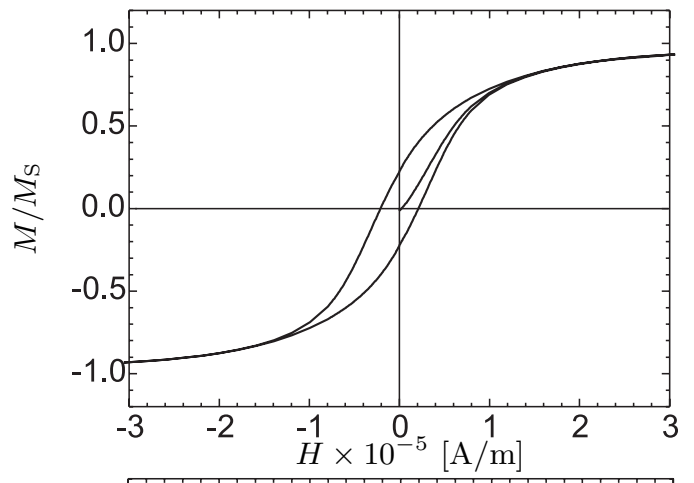

(a)

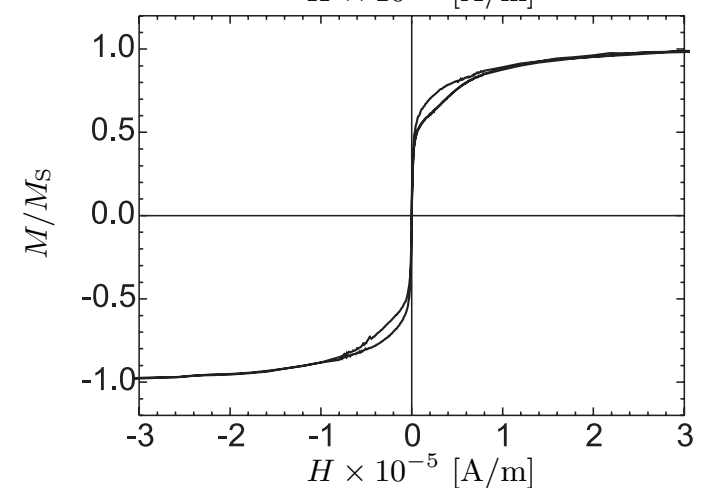

(b)

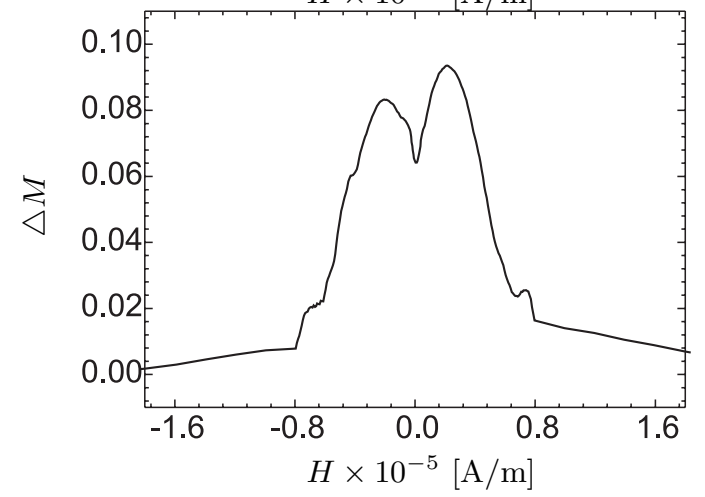

(c)

Fig. 5. (a) Quasi-static magnetization curve of Co-0T powder; (b) of Co-0T dispersion; (c) variance graph $\left(M_{\mathrm{up}}-M_{\mathrm{down}}\right)$ for Co-0T dispersion in toluene.

volume-average magnetic core size $d_{\mathrm{m}}$, accessible at magnetogranulometry by assuming the cores are pure cobalt $\left(M_{\mathrm{S}}=1.46 \cdot 10^{6} \mathrm{~A} / \mathrm{m}\right)$, results in similar values around $13 \mathrm{~nm}$. This is in contrast to the TEM images, which indicate a variation of the core size with the TOPO content. It is in accordance, however, with the decrease in saturation magnetization of the powders with the rising TOPO coordination, probably indicating the formation of a distinct magnetically dead layer on the core surface.

The results indicate that the contribution of TOPO to the observed structure formation is threefold: as it can be observed, the Co cores' increase of core diameter in the presence of TOPO during synthesis does not result in a higher magnetic moment of the core, instead, the formation of an oxidic layer with lower or no magnetization is supposed. In addition, the presence of coordinating TOPO on the particle surface can lead to surface charges, as indicated in Fig. 1. The resulting 
Table 3. Magnetic properties of Co@PS/TOPO particle dispersions in toluene. $M_{\mathrm{S}}-$ saturation magnetization, $\chi_{\mathrm{ini}}$ - initial susceptibility, $\mu$ - particle magnetic moment, $d_{\mathrm{m}}$ - volume average diameter by VSM, $\sigma$ - standard deviation.

\begin{tabular}{ccccccc}
\hline \hline $\begin{array}{c}\text { Sample } \\
\text { [liquid }]\end{array}$ & $\begin{array}{c}c_{\mathrm{TOPO}} \\
{[\mathrm{mmol} / \mathrm{l}]}\end{array}$ & $\begin{array}{c}M_{\mathrm{S}} \\
{[\mathrm{A} / \mathrm{m}]}\end{array}$ & $\chi_{\text {ini }} / M_{\mathrm{S}}$ & $\begin{array}{c}\mu \\
{[\mathrm{A} / \mathrm{m}]}\end{array}$ & $\begin{array}{c}d_{\mathrm{m}} \\
{[\mathrm{nm}]}\end{array}$ & $\sigma$ \\
\hline Co-0T & 0 & $1.74 \cdot 10^{3}$ & $2.21 \cdot 10^{-4}$ & $2.20 \cdot 10^{-18}$ & 13.6 & 0.71 \\
Co-6.3 & 6.3 & $4.88 \cdot 10^{2}$ & $1.68 \cdot 10^{-4}$ & $1.67 \cdot 10^{-18}$ & 13.0 & 0.61 \\
$\mathrm{Co}-12.7 \mathrm{~T}$ & 12.7 & $4.27 \cdot 10^{2}$ & $1.90 \cdot 10^{-4}$ & $1.83 \cdot 10^{-18}$ & 13.2 & 0.41 \\
$\mathrm{Co}-25.4 \mathrm{~T}$ & 25.4 & $1.3 \cdot 10^{3}$ & $1.9 \cdot 10^{-4}$ & $1.9 \cdot 10^{-18}$ & 12.8 & 0.35 \\
\hline \hline
\end{tabular}

electrostatic repulsion is hardly screened from ions in the medium, as the ionic strength in the organic toluene-based dispersion can be assumed low.

The surface charge density will be investigated in zeta potential experiments in ongoing experiments in order to verify this hypothesis.

4. Conclusions. The use of TOPO as a co-stabilizer in the synthesis of Co@PS/TOPO nanoparticles strongly influences the particle interaction potential in dispersion and as a powder, as evidenced by VSM, AC susceptometry and DLS. The attractive and repulsive forces contribute to the particle interaction and to the superstructure formation on the tens of the nanometer scale, and contribute to a trend in the ability to form extended chains in dispersion or upon drop-casting. These observations can be explained by the presence of a surface charge generated by the coordination of polar TOPO on the particle surface.

This interplay of repulsive electrostatic and attractive dipole-dipole forces among the particles opens a new pathway of tailoring super-structures of ferromagnetic particles.

\section{REFERENCES}

[1] C.A. Mirkin, R.L. Letsinger, R.C. Mucic, J.J. Storhoff. Nature, 382 (1996), pp. 607-609.

[2] N.L. Rosi And C.A. Mirkin. Chem. Rev., 105 (2005), pp. 1547-1562.

[3] Y. Lin, A. Boker, J. He, K. Sill, H. Xiang, C. Abetz, X. Li, J. Wang, T. Emrick, S. Long, Q. Wang, A. Balazs, T.P. Russell. Nature, 434 (2005), pp. 55-59.

[4] C. Mao, D.J.Solis, B.D. Reiss, S.T.Kottmann, R.Y.Sweeney, A. Hayhurst, G. Georgiou, B. Iverson and A.M. Belcher. Science, 303 (2004), pp. 213-217.

[5] M.J. Misner, H. Skaff, T. Emrick, T.P. Russell. Advanced Mater, 15 (2003), pp. 221-224.

[6] M.R. Bockstaller, R.A. Mickiewicz, E.L. Thomas. Advanced Mater, 17 (2005), pp. 1331-1349.

[7] S.-W. Lee, C. MaO, C.E. Flynn, A.M. Belcher. Science, 296 (2002), pp. $892-895$.

[8] J.C. HuIE. Smart Mater. Struct, 12 (2003), p. 264. 
C.Dobbrow, M. Vaidyanathan, L. Belkoura, D. Guin, A.M. Schmidt

[9] P.Y. Keng, I. Shim, B.D. Korth, J.F. Douglas, J. Pyun. ACS Nano, 1 (2007), pp. 279-292.

[10] J.J. Benkoski，S.E. Bowles， B.D. Korth， R.L. Jones，J.F. Douglas, A. Karim, J. Pyun. J. Am. Chem. Soc, 129 (2007), pp. 6291-6297.

[11] A.P. Philipse, D. MaAs. Langmuir, 18 (2002), pp. 9977-9984.

[12] M. Klokkenburg, C. Vonk, E.M. Claesson, J.D. Meeldijk, B.H. Erne, A.P. Philipse. J. Am. Chem. Soc, 126 (2004), pp. 16706-16707.

[13] M.-J. Hu, Y.Lu, S.Zhang, S.-R. Guo, B. Lin, M. Zhang, S.-H. Yu. J. Am. Chem. Soc, 130 (2008), pp. 11606-11607.

[14] H. Wang, Q.-W. Chen, Y.-B. Sun, M.-S. Wang, L.-X. Sun, W.-S. Yan. Langmuir, 26 (2010), pp. 5957-5962.

Received 03.07.11. 